\title{
ELECTROMAGNETIC CHARACTERIZATION OF METALLIC SENSORY ALLOY
}

\author{
Buzz Wincheski ${ }^{1}$, John Simpson ${ }^{2}$, Terryl Wallace ${ }^{1}$, Andy Newman ${ }^{1}$, Paul Leser ${ }^{3}$, and Rob \\ Lahue $^{4}$ \\ ${ }^{1}$ NASA Langley Research Center, Hampton, VA 23681 \\ ${ }^{2}$ Northrop Grumman, Hampton, VA 23681 \\ ${ }^{3}$ North Carolina State University, Raleigh, NC 27695 \\ ${ }^{4}$ Elon University, Elon, NC 27244
}

\begin{abstract}
Ferromagnetic shape-memory alloy (FSMA) particles undergo changes in both electromagnetic properties and crystallographic structure when strained. When embedded in a structural material, these attributes can provide sensory output of the strain state of the structure. In this work, a detailed characterization of the electromagnetic properties of a FSMA under development for sensory applications is performed. In addition, a new eddy current probe is used to interrogate the electromagnetic properties of individual FSMA particles embedded in the sensory alloy during controlled fatigue tests on the multifunctional material.
\end{abstract}

Keywords: Ferromagnetic Shape Memory Alloy, Eddy Current, Strain Sensing

PACS: 81.70.Ex, 81.05.Bx, 81.30.Kf

\section{INTRODUCTION}

Recent work at NASA Langley Research Center has investigated the potential of a new multifunctional structural metallic material containing second phase sensory particles. Ferromagnetic shape-memory alloy (FSMA) particles embedded in an aluminum matrix undergo changes in both crystallographic structure and electromagnetic properties when strained. The crystallographic changes result in high-energy acoustic emissions, while the electromagnetic changes can lead to enhanced detection and localization of high strain areas associated with the plastic deformation leading a crack front. In this work, a detailed characterization of the electromagnetic properties of the FSMA is performed. In addition, a new electromagnetic probe consisting of a two-channel magneto-resistive sensor with an embedded single-strand eddy current inducer is used to probe the electromagnetic properties of individual FSMA particles embedded in the sensory alloy during controlled fatigue tests on the multifunctional material. The probe is used in both high-frequency eddy current and DC flux leakage applications to characterize the changes in the permeability and conductivity of the sensory particles due to the strain field of an approaching fatigue crack. 


\section{ELECTROMAGNETIC CHARACTERIZATION OF FERROMAGNETIC SHAPE MEMORY ALLOYS FOR SENSORY APPLICATIONS}

Ferromagnetic shape memory alloys undergo a martensitic transformation in response to temperature, strain, or external magnetic field [1-3]. While much previous work has focused on applications of the material for the development of mini-actuators [4], sensory applications of the material also have been suggested in the literature [5]. As a sensory material, a strain-induced martensitic transformation could be detected nondestructively by the associated changes in the electromagnetic properties of the alloy. Under optimal conditions external strain is predicted to induce a paramagnetic to ferromagnetic transition such that magnetization measurements could be used to measure strain. As details of the fabrication of the FSMA being developed at NASA LaRC have been previously reported [6], this work will focus on characterization of the electromagnetic properties of the material.

The magnetic properties of the FSMA as a function of temperature and magnetic field were studied using a vibrating sample magnetometer equipped with a liquid nitrogen cooled dewar. Magnetization curves were acquired at temperatures between 100 and 375 Kelvin and applied fields in the range of $+/-5 \mathrm{kOe}$. Fig. 1 displays the results for magnetization curves acquired at 100, 200, and 300 Kelvin, with the inset displaying the measured coercivity as a function of temperature. As the temperature is decreased a dramatic increase in both the coercivity and saturation magnetization is observed, consistent with a paramagnetic to ferromagnetic transition.

The temperature dependence of the magnetization and temperature derivative of the magnetization are plotted in Fig. 2. For these data the temperature was set at 100 Kelvin and then ramped up to $375 \mathrm{~K}$ and back to $100 \mathrm{~K}$ with the applied field held constant at 500

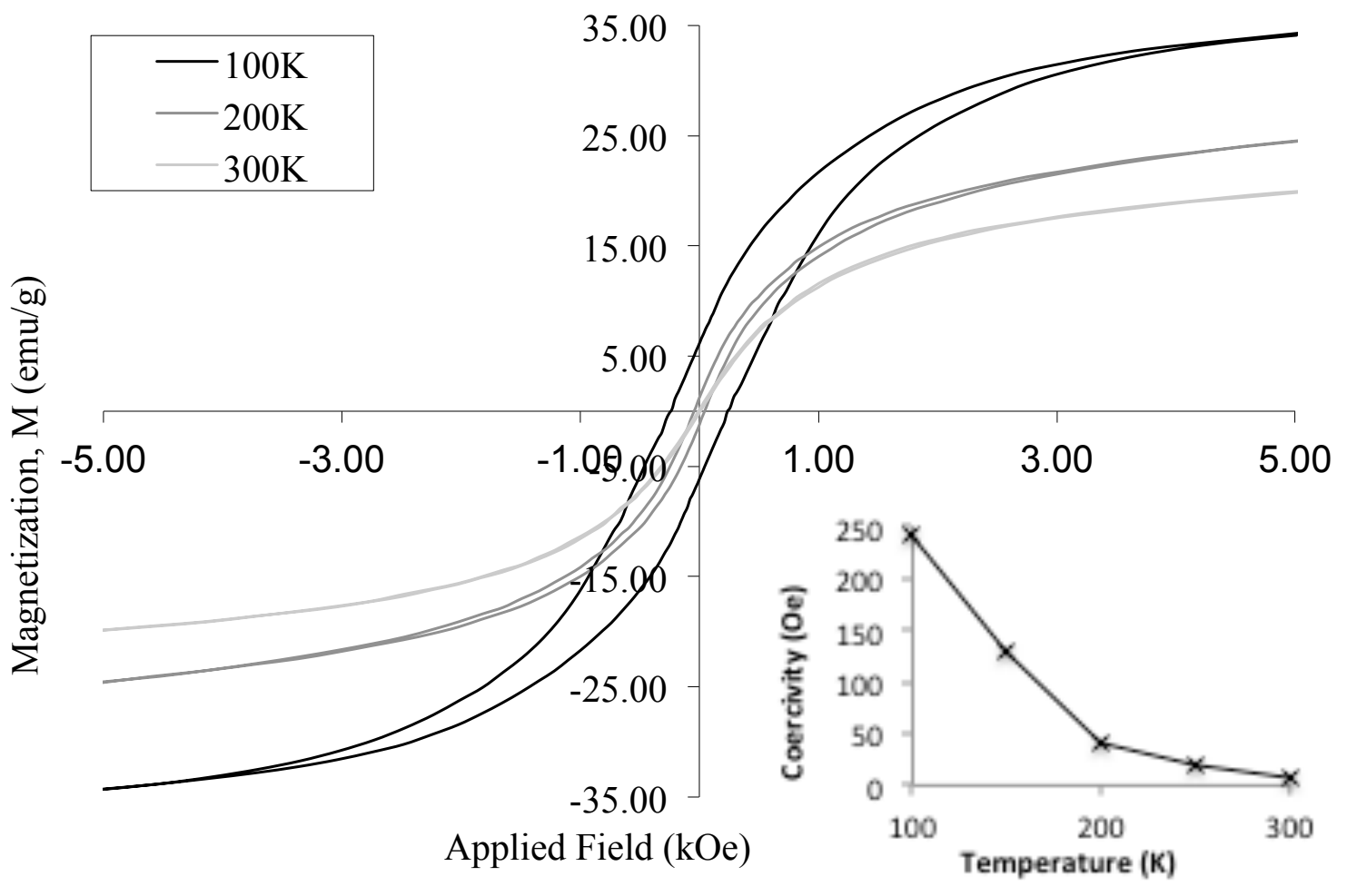

FIGURE 1. Magnetization data for FSMA as measured by vibrating sample magnetometer. Material undergoes ferromagnetic to paramagnetic transition within this temperature range. 


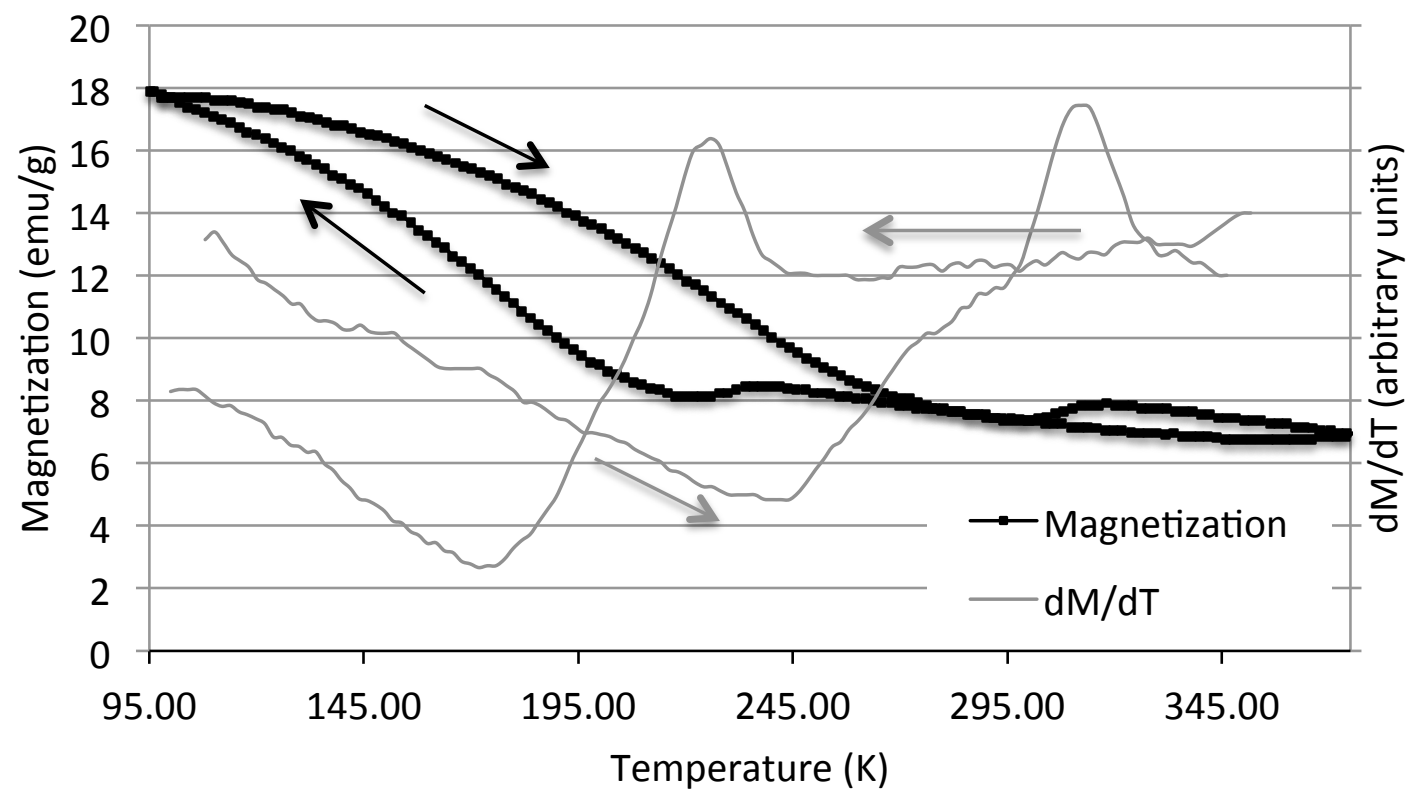

FIGURE 2. Sample magnetization and temperature derivative of magnetization versus temperature at 500 Oe applied field.

Oe. Magnetization measurements were acquired with a vibrating sample magnetometer at 2 Kelvin increments. A clear hysteresis in the data is observed and can be explained based upon the Curie temperature $\left(\mathrm{T}_{\mathrm{c}}\right)$ and phase transformation between martensitic and austenitic states. The alloy starts in the low temperature ferromagnetic martensitic state. At approximately $240 \mathrm{~K}$ the martensitic $\mathrm{T}_{\mathrm{c}}$ is reached, indicated by the local minimum point in the plot of the derivative of magnetization $(\mathrm{dM} / \mathrm{dT})$ versus temperature $[5,7]$. The temperature of the now paramagnetic martensitic alloy continues to increase to approximately $310 \mathrm{~K}$ where an increase in the magnetization and an associated local maximum in the plot of $\mathrm{dM} / \mathrm{dT}$ is observed. This step in the M-T curve marks the austenitic transition of the alloy [5]. Above $330 \mathrm{~K}$ the alloy is in a stable paramagnetic austenitic phase. Upon cooling, the alloy remains in the paramagnetic austenitic phase below the martensitic $T_{c}$ near $240 \mathrm{~K}$. This shows that the Curie temperature of the martensitic phase is higher than that of the austenitic phase. A strain-induced martensitic transformation of the paramagnetic austenitic alloy at a temperature below $240 \mathrm{~K}$ would then result in a ferromagnetic martensitic phase. Upon continued cooling the martensitic transformation is observed near $225 \mathrm{~K}$. The martensitic alloy, now below the Curie temperature, transitions to the ferromagnetic phase as indicated by the rapid increase in magnetization.

Along with changes in the magnetic properties of the FSMA, changes in the resistivity also are anticipated between the martensitic and austenitic phases [3]. To track this effect in the alloys under investigation at NASA LaRC, temperature-dependent resistivity measurements were performed. A Lakeshore $370 \mathrm{AC}$ resistance bridge was used in a four-lead configuration to measure the resistance of the samples as a function of temperature with the samples mounted in a liquid nitrogen cooled cryostat. Fig. 3. displays the normalized change in resistance of the FSMA as the temperature is varied from $360 \mathrm{~K}$ to $175 \mathrm{~K}$ and back to $360 \mathrm{~K}$. Solid lines have been overlaid on the data to aid the eye in interpretation of the data. A sharp increase in resistance is measured beginning at $\sim 275 \mathrm{~K}$ and continuing to $\sim 210 \mathrm{~K}$, marking the start and finish temperatures of the martensitic transformation. The austenitic transformation temperatures can likewise be interpreted as $265 \mathrm{~K}-315 \mathrm{~K}$. Note that these electrical measurements of transformation temperatures are 


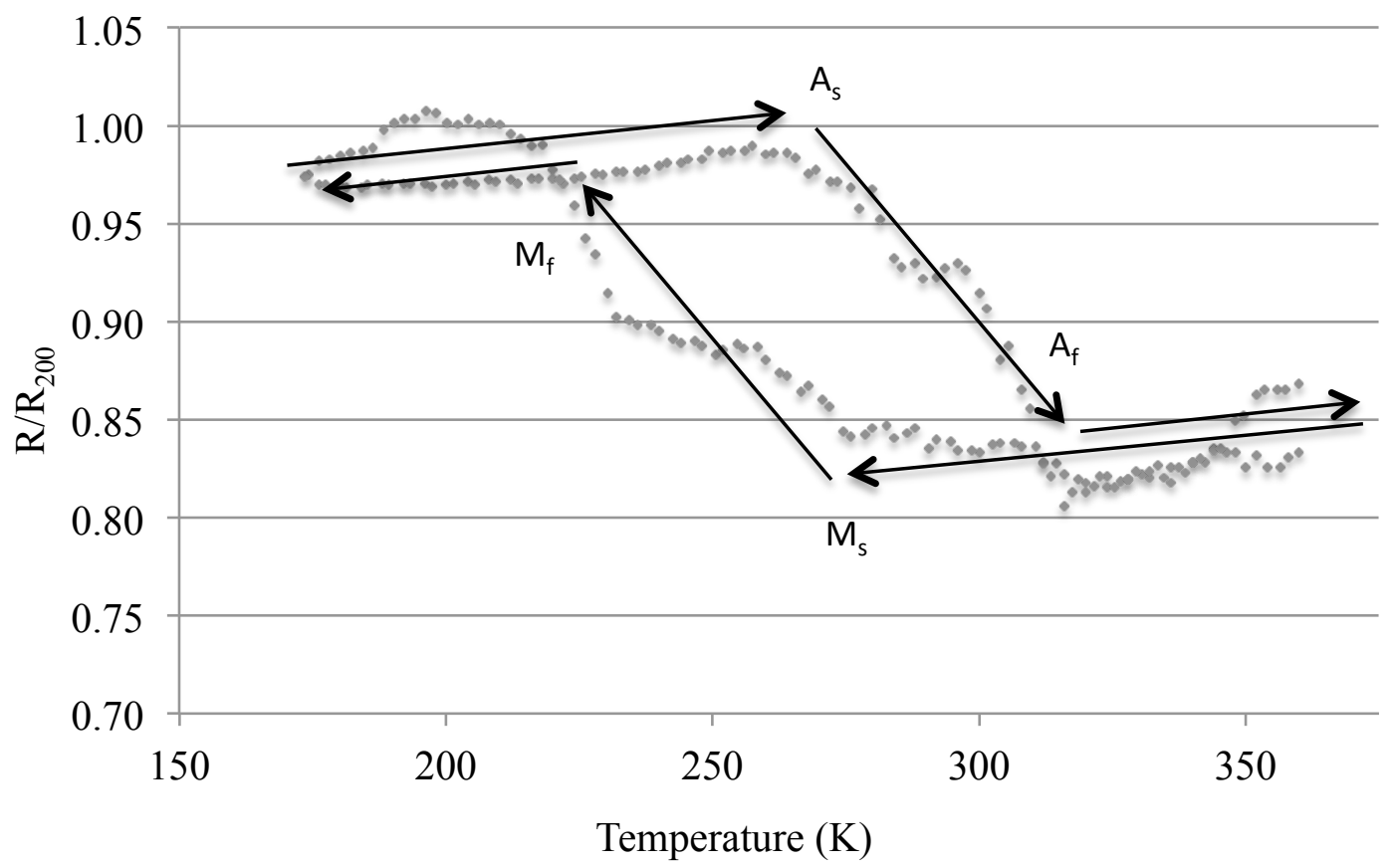

FIGURE 3. FSMA resistance as a function of temperature normalized to resistance at $200 \mathrm{~K}$.

in good agreement with the magnetically measured temperatures at $310 \mathrm{~K}(225 \mathrm{~K})$ for the austenitic (martensitic) transformations. In agreement with [3], an increase in resistance of $\sim 15 \%-20 \%$ is measured across the austenitic - martensitic transformation, with an equivalent decrease in resistance during the reverse transformation. A strain-induced martensitic transformation would therefore be anticipated to produce an increase in the resistivity of the sample on the order of $15 \%-20 \%$.

\section{SENSORY ALLOY ANALYSIS}

As discussed in the previous section, the martensitic transformation of a FSMA results in measurable changes to both the magnetic and electrical properties of the material. Work at NASA LaRC seeks to capitalize on these physical properties to enable nondestructive and noncontact strain measurement of aerospace alloys and structures. Initial work has shown that sensory alloys produced through integration of FSMA particles into an aluminum matrix have no apparent decrease in fatigue life as compared to panels without particles [6], and that high-resolution eddy-current imaging can isolate individual particles in a sensory alloy [8]. The research focus has now turned to measuring the electromagnetic properties of individual FSMA particles embedded in the sensory alloy as a means to investigate material strain. This effort has focused on application of the highresolution magneto-resistive sensor based eddy current probe described in [8].

As discussed in [8], the high-resolution magneto-resistive based eddy current probe utilizes a single inducer wire embedded into the face of a two-axis anisotropic magnetoresistive (AMR) sensor. In this work a slight redesign of the inducer wire has been incorporated to minimize the elongation of features along the axis of the inducer wire, allowing better localization of anisotropic features in the sample. In addition, DC operation of the probe has been utilized to probe the stray magnetization of the FSMA particles.

Fig. 4. displays $\mathrm{C}$-scan images of both the induced eddy current signature and the stray magnetic field for a sensory alloy sample with embedded FSMA particles. The highfrequency eddy current image highlights near surface particles due to the lower 
EC Amp. (V)
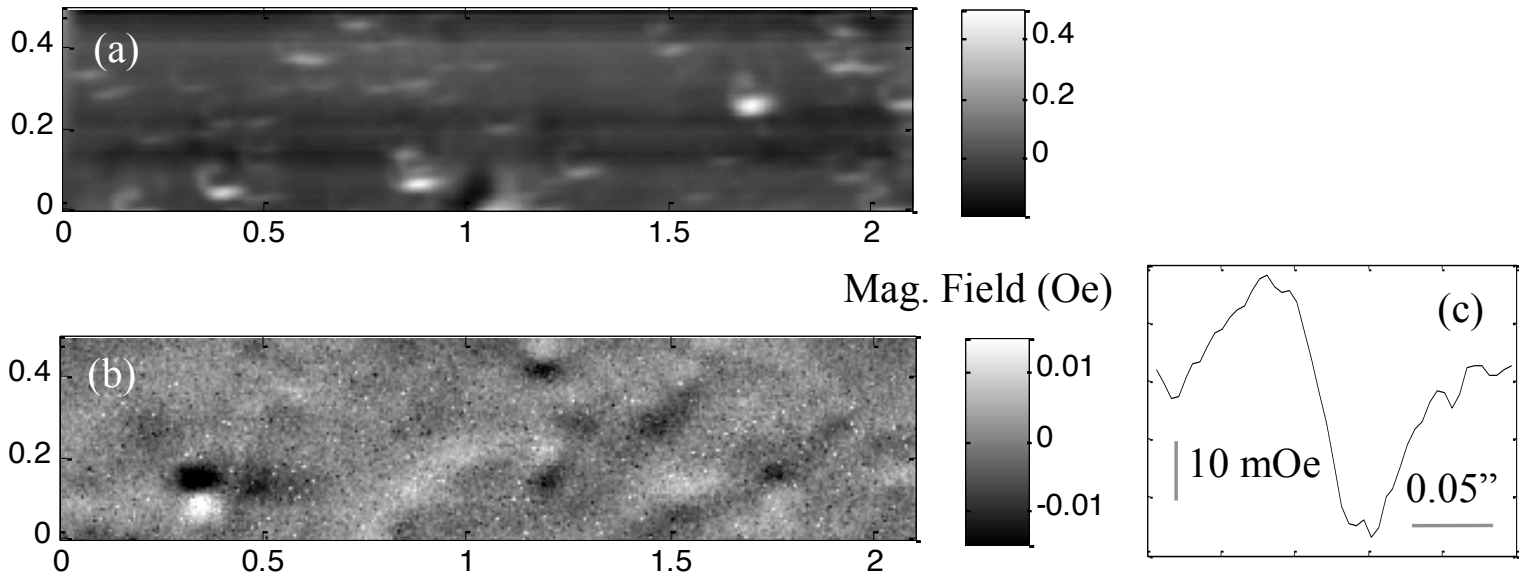

FIGURE 4. (a) $1 \mathrm{MHz}$ eddy current and (b) DC stray magnetic field images of sensory alloy with embedded FSMA particles. (c) Vertical line scan of DC magnetic field across particle located at $(.335,0.125)$. All dimensions are in inches.

conductivity and higher permeability of the FSMA particles compared to the aluminum matrix while the DC magnetic field map localizes particles with the strongest residual magnetization. Both images in Fig. 4 are acquired by measuring the magnetic field at the sample surface in the direction of the short axis of the sample.

Eddy current data are acquired using an induction current at $1 \mathrm{MHz}$ drive frequency to induce eddy currents in the sample while the magnetic field amplitude and phase at 1 $\mathrm{MHz}$ are monitored using a lock-in amplifier. Fig. 4a is the C-Scan for the magnitude of the magnetic field at $1 \mathrm{MHz}$. Both the lower conductivity and higher permeability of the FSMA particles as compared to the aluminum matrix lead to an increase in the measured field level when the probe is located above a FSMA particle. The reduced conductivity results in a lower eddy current intensity at the location of the particles. The resultant field level at the sensor location is increased as the eddy currents are less effective at countering the drive field produced by the induction wire. The increased permeability results in a low reluctance path for the magnetic field, increasing the field level at the sensor location.

Stray magnetic field data are acquired with the induction current turned off. The DC output of the sensor is monitored to image the static magnetic field across the sample surface. The magnetizable FSMA particles produce a localized magnetic field, as shown in Fig. 4b. Fig. 4c highlights the stray magnetization of a particle located at (0.335", 0.125 "). As the sensor is measuring fields in the vertical direction, the dipolar pattern corresponds to a particle at this location with a net magnetization into the page. DC magnetization imaging of the stray field in the horizontal direction confirms this analysis.

\section{IN-SITU TESTING}

Following the successful imaging of the FSMA alloy particles in the sensory alloy, a fatigue test of the material was designed in which eddy current and stray magnetic field imaging could be performed at intermediate steps in the fatigue process. Fig. 5 displays a picture of the experimental setup along with a close up view of a typical sensory alloy. In the image the eddy current probe is removed from the surface. During fatigue cycling a high-magnification optical microscopy focused on the crack tip, the compliance of the sample, and acoustic emission events were analyzed to track any growth in the fatigue crack. At signs of crack extension the fatigue process was stopped and high-resolution eddy current and DC magnetization data were acquired with the AMR based probe. Eddy 
current and DC magnetization data were acquired using a motion control system and spring-loaded probe carrier to maintain uniform contact between the probe and the sample surface. Along with the AMR probe and sensory alloy, magnetizing coils are visible in the image. The coils were used in an attempt to demagnetize the load frame grips and measure the stray magnetization of the FSMA particles as a function of applied field. Unfortunately the field strength was not high enough to demagnetize the grips and the residual magnetization of the grips obscured small changes in the static field distribution due to the localized FSMA particles.

Fig. 6 displays the high-frequency C-Scan of a sensory alloy that was acquired with the system setup as shown in Fig. 5. The sample contained a starter notch at the left edge of the sample, similar to that shown in the close up of the sensory alloy sample in Fig. 5. Slightly ahead of the notch a FSMA particle could be visibly detected and was also readily imaged with the high-frequency mode of the AMR high-resolution probe. Other FSMA particles were also imaged in the sample as regions with increased response. In particular, a strong indication was recorded for a particle slightly above the notch location. The sensory alloy from this test was imaged at three different fatigue intervals, starting with a baseline scan in the load frame. Data analysis consisted of measuring the response of the FSMA particle preceding the crack and normalizing this amplitude to the particle outside of the crack growth direction. Also shown in Fig. 6 is a line scan of the probe response across the indicator particle, weighted by the probe response to the normalization particle. The line scan across the particle after the second fatigue run is virtually identical to the baseline data. After the third fatigue cycle run, however, an increase in the probe response of over ten percent is detected. While not conclusive, the results suggest that strain in the sample preceding the crack tip may have induced an austenitic to martensitic transformation in the indicator particle. The associated increase in resistivity in the martensitic state would then account for the increased probe response at the location of the particle.

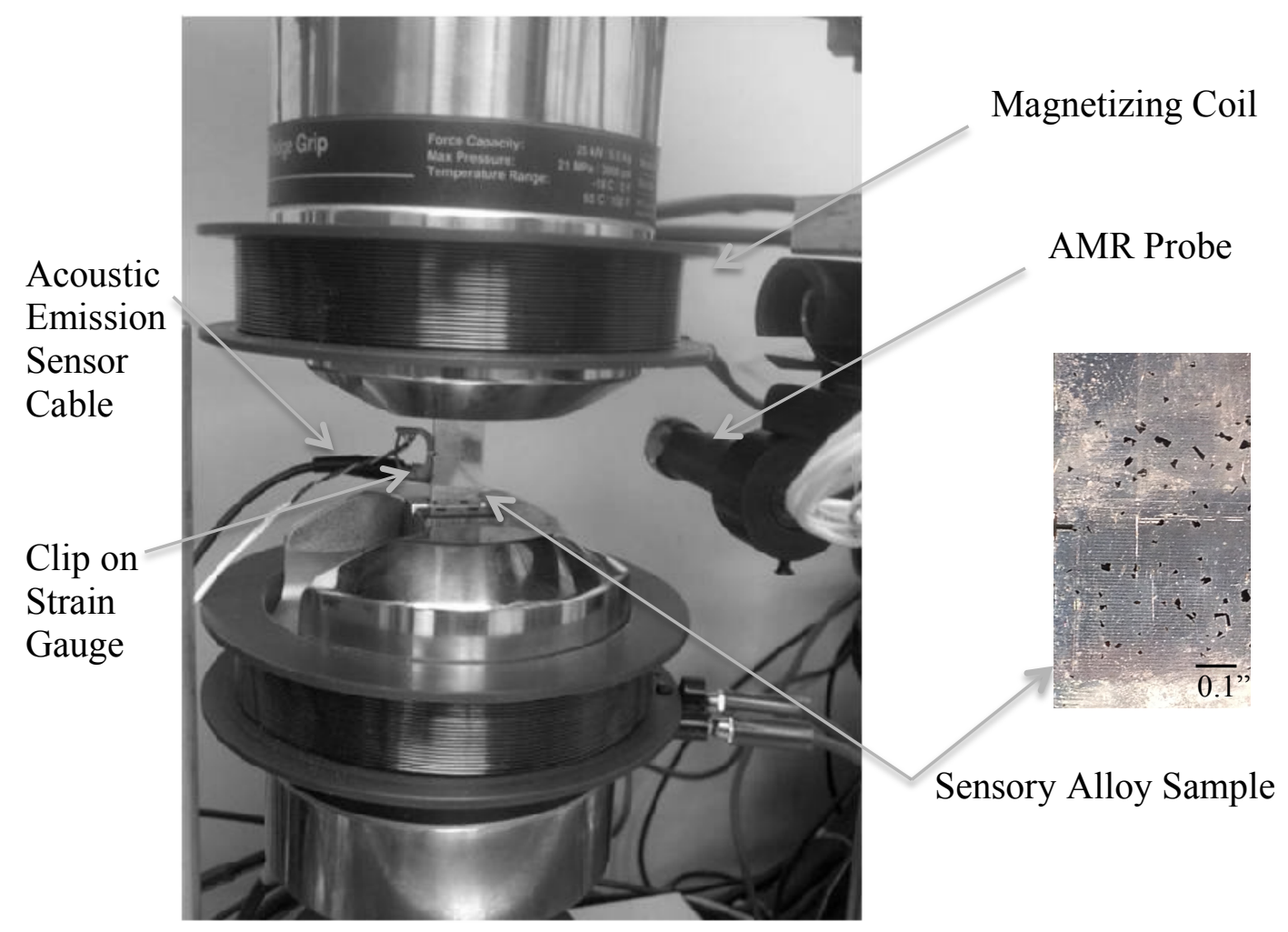

FIGURE 5. Experimental setup and close up view of sensory alloy mounted in fatigue test equipment. 


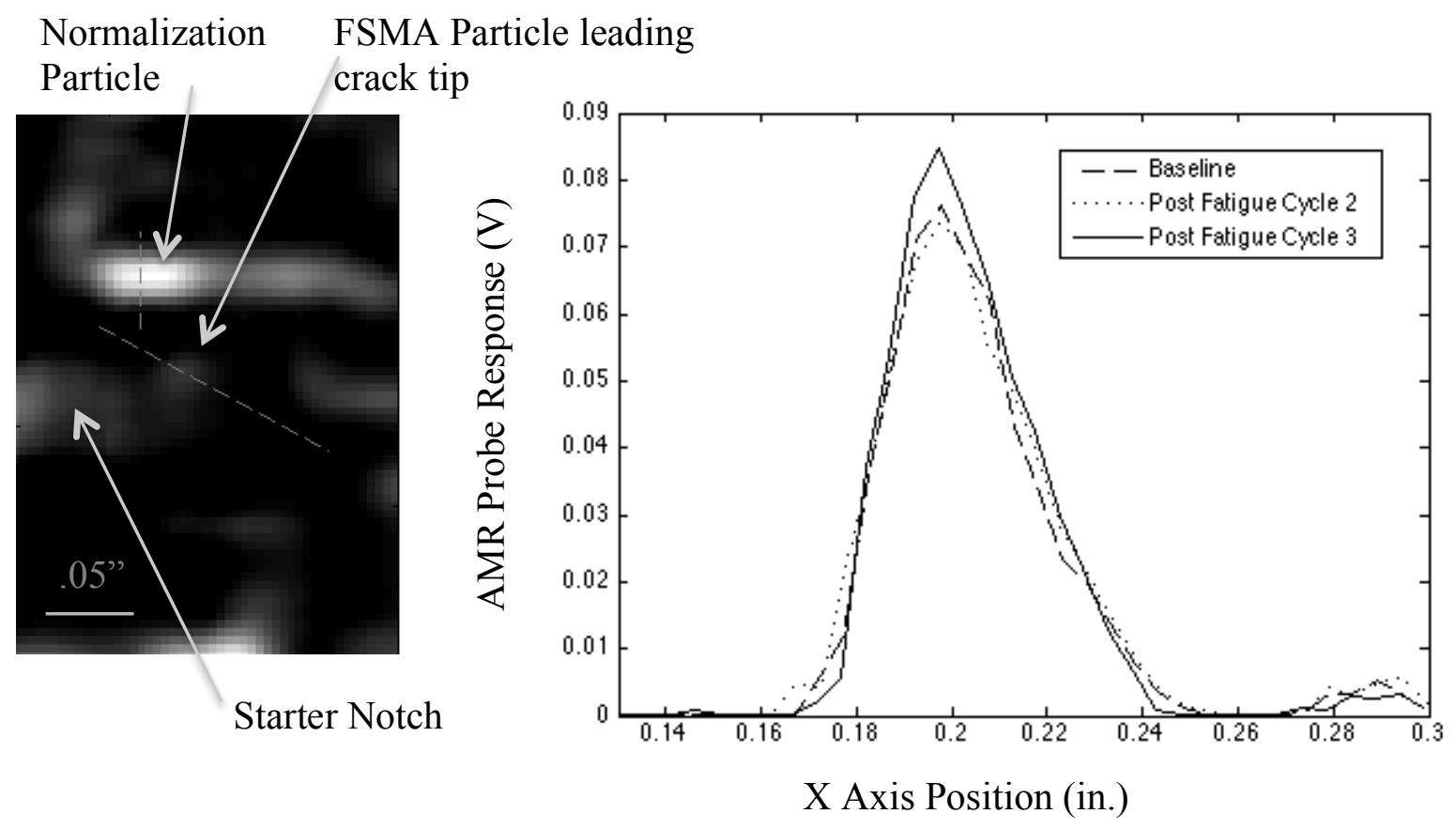

FIGURE 6. High-frequency AMR probe C-Scan performed on sample in load frame and line scans across particle proceeding crack at three different fatigue cycle intervals.

\section{SUMMARY}

In this work we have investigated the electromagnetic properties of a ferromagnetic shape memory alloy that is being studied for use in sensory material development. Analyses of the electromagnetic properties have shown that the FSMA undergoes an austenitic to martensitic transition below room temperature, such that the alloy cooled alloy from high temperature will be in a stable austenitic state at room temperature. Analyses of the magnetic properties have also shown that the Curie temperature of the martensitic phase exceeds that of the austenitic phase. This important attribute opens the possibility for a strain-induced transformation from a paramagnetic austenitic phase to a ferromagnetic martensitic phase. In addition, temperature-dependent conductivity measurements of the FSMA have shown that the martensitic transition is associated with an increased resistivity of $15-20 \%$.

The second phase of this work investigated the potential for monitoring individual FSMA particles in a sensory alloy as a means to detect material strain. An anisotropic magneto-resistive sensor based probe was used to study both the high-frequency eddy current and DC magnetic properties of individual FSMA particles. Material strain is anticipated to induce an austenitic to martensitic transformation in the particles that can then be detected by changes in the conductivity and permeability of the material. An insitu application of the probe during a high cycle fatigue test of a sensory alloy was performed and showed promising results in detecting changes to the electromagnetic properties of the FSMA particles due to material strain. Work in this area is proceeding with continued in-situ monitoring of sensory alloys during controlled fatigue tests as well as with further FSMA particle optimization. These optimization studies will be designed to produce FSMA particles that show that greatest change in magnetic and electrical properties due to a strain-induced martensitic transformation at room temperature. 


\section{REFERENCES}

1. V.A. Chernenko and S. Besseghini, "Ferromagnetic shape memory alloys: Scientific and applied aspects, ”Sens. Actuators A, Vol. 142, pp. 542-548, 2008.

2. R. Kainuma, Y. Imano, W. Ito, Y. Sutou, H. Morito, S. Okamoto, O. Kitakami, K. Oikawa, A. Fujita, T. Kanomata, and K. Ishida, "Magnetic-field-induced shape recovery by reverse phase transformation," Nature, Vol. 439, pp. 957 - 960, 2006.

3. I.I. Kositsyna, and V.A. Zavalishin, "Study of Co-Ni-Al Alloys with Magnetically Controlled Shape Memory Effect," Materials Science Forum, Vol. 635, pp. 75 - 80, 2010.

4. A. Nespoli, S. Besseghini, S. Pittaccio, E. Villa, and S. Viscuso, "The high potential of shape memory alloys in developing miniature mechanical devices: A review on shape memory alloy mini-actuators," Sens. Actuators A, Vol. 158, pp. 149-160, 2010.

5. K. Oikawa, L. Wulff, T. Iijima, F. Gejima, and T. Ohmori et. al., "Promising ferromagnetic Ni-Co-Al shape memory alloy system," Appl. Phys. Lett., Vol. 79, pp. $3290-3292,2001$.

6. T. Wallace, W. Leser J. Newman, and P. Leser, "Development of Metallic Sensory Alloys," Presented at 2012 Aircraft Airworthiness \& Sustainment Conference, Baltimore, MD, 2012.

7. H. Saito, T. Yokoyama, K. Kiamichi, K. Kamishima, and T. Goto, "Itinerant-electron metamagnetism of the Laves-phase compounds $\mathrm{Lu}\left(\mathrm{Co}_{1-\mathrm{x}} \mathrm{Ga}\right) 2$ under high pressures with high magnetic fields', Phys. Rev. B., Vol. 59, pp. 8725-8731, 1999.

8. B. Wincheski and J. Simpson, J.P. Seebo, and J. Powell, "High resolution imaging with two-axis orthogonal magneto-resistive sensor based eddy current probe," in Review of Progress in Quantitative NDE, Vol. 31, pp. 366-372, 2012. 